\title{
Collaborative Coverage for a Network of Vacuum Cleaner Robots
}

DOI:

10.1007/978-3-030-89177-0_11

\section{Document Version}

Accepted author manuscript

Link to publication record in Manchester Research Explorer

\section{Citation for published version (APA):}

Hu, J., Lennox, B., \& Arvin, F. (2021). Collaborative Coverage for a Network of Vacuum Cleaner Robots. In 2021 Annual Conference Towards Autonomous Robotic Systems https://doi.org/10.1007/978-3-030-89177-0_11

\section{Published in:}

2021 Annual Conference Towards Autonomous Robotic Systems

\section{Citing this paper}

Please note that where the full-text provided on Manchester Research Explorer is the Author Accepted Manuscript or Proof version this may differ from the final Published version. If citing, it is advised that you check and use the publisher's definitive version.

\section{General rights}

Copyright and moral rights for the publications made accessible in the Research Explorer are retained by the authors and/or other copyright owners and it is a condition of accessing publications that users recognise and abide by the legal requirements associated with these rights.

\section{Takedown policy}

If you believe that this document breaches copyright please refer to the University of Manchester's Takedown Procedures [http://man.ac.uk/04Y6Bo] or contact uml.scholarlycommunications@manchester.ac.uk providing relevant details, so we can investigate your claim.

\section{OPEN ACCESS}




\title{
Collaborative Coverage for a Network of Vacuum Cleaner Robots ${ }^{\star}$
}

\author{
Junyan $\mathrm{Hu}^{1}$, Barry Lennox ${ }^{1}$, and Farshad Arvin ${ }^{1}$ \\ University of Manchester, Manchester, UK \\ \{junyan.hu, barry.lennox, farshad.arvin\}@manchester.ac.uk
}

\begin{abstract}
Coordination of mobile robot teams has attracted significant attention in the area of robotics research. As one of the most important techniques used in the multi-robot systems, coverage has shown great potential to be applied to many real-world applications. In this paper, we aim to provide a novel path planning method for multi-robot coverage with applications to cooperative autonomous vacuum cleaning. Some preliminary results are presented using an open-source simulator Webots, which lay the foundation for more in-depth theoretical analysis and practical implementation in the subsequent research.
\end{abstract}

Keywords: Coverage · Navigation · Path Planning · Swarm Robotics.

\section{Introduction}

Autonomous vacuum cleaning, as one of the most successful applications of mobile service robotics, has received significant attentions since the past decade. Simultaneous localization and mapping techniques are mostly used in the robotic platforms, which lead to reliable coverage performances in small-scale environments. However, with the increasing demand in using robot swarms to collaboratively clean a common large-scale area like hotels, warehouses, office buildings, etc., high-efficiency coordination algorithms for networked cleaner robots are being explored by researchers from both academia and industry.

A team of vacuum cleaner robots can be viewed as a multi-robot system, which shows great potential to be used in real-world applications due to its flexibility, reconfigurability, robustness to faults and cost-effectiveness in solving complex tasks. Some potential applications of multi-robot teams include cooperative transportation [1,2], target monitoring [3], etc. When properly designed, a multi-robot system can provide a more efficient and robust performance compared to a single robot [4]. Various of coordination strategies have been developed by researchers in recent years. Some main research directions include collective decision making, swarm intelligence, multi-robot path planning, formation control, etc. Consider the features of the cooperative cleaning tasks, coverage techniques can be utilized to fulfil the objective.

\footnotetext{
* This work was supported in part by EPSRC-IAA and TPLC ltd, in part by EPSRC RAIN and RNE projects [EP/R026084/1 and EP/P01366X/1], and in part by the Royal Academy of Engineering [grant number CiET1819].
} 
To achieve the goal, robots should be able to communicate with each other to obtain relative position information via a decentralized network. Besides, a control structure should also be designed properly to ensure the robots achieve the pre-specified goal by using the information obtained from the sensors and communication modules. In this paper, we aim to provide a novel coordination solution for multi-robot collaborative cleaning problems.

\section{Method}

Each vacuum cleaner robot can be viewed as a cyber-physical system. We implement a two-layer control structure for the robot teams, which consists of a cyber layer for the decision making purpose and a physical layer for the target tracking purpose.

In the cyber layer, a frontier-based exploration technique [5] is mainly used to achieve autonomous coverage, where the radius of the frontier point detection should be set the same as the robot's radius to ensure that the robot's footprints fully cover the explored area. To avoid repeated trajectories and overlapping cleaning areas when using multiple robots in the collaborative task, a Voronoi-based path planning technique [6] is also added to improve efficiency. By using the relative position information from the neighbors via the connected communication network, some Voronoi partitions are generated automatically, thus each robot will give a higher priority to the target area in its local Voronoi cell before moving to other robots' working zones. Combine these two decision making processes, a desired set of waypoints can be generated, which are then transmitted to the physical layer for target tracking.

In the physical layer, the assigned waypoint should be tracked precisely by implementing a robust control system based on the robot dynamics. Firstly, the nonlinear robot dynamic model is transformed to a linear system using an inputoutput feedback linearization controller [7]. Then an adaptive tracking controller which was proposed in [8] can be applied to the robot to achieve position tracking using the state feedback from the onboard sensors.

The proposed coverage strategy will terminate only if there is not new frontier point in the whole environment, which means the working area is fully covered by the robots' footprints.

\section{$3 \quad$ Results}

In this section, Webots [9] is selected as the simulation platform, which provides a realistic environment to test the effectiveness of the theoretical results. As a professional robotic platform, Webots has integrated cross-compilation systems allowing users to compile and upload the controllers to real robots with minimum modification, which facilitates the real-robot application of the proposed control architecture.

In the simulation, the arena's size is set as $6 \mathrm{~m} \times 6 \mathrm{~m}$. We use five vacuum cleaner robots to perform the collaborative cleaning task as shown in Fig. 1. For 


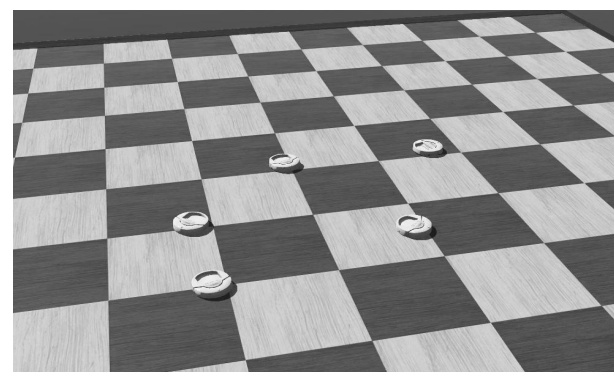

Fig. 1. Five cleaner robots are used in the simulation case study.

comparison, three different methods are applied to the robots during the mission. Firstly, a default control algorithm is directly implemented in the robotic platform, which reflects the random movements commonly observed in the early version of the cleaner robot products. Based on the proposed coverage strategy, a semi-coordinated algorithm is also tested, where the robots are able to cooperate with each other to cover the working area via Voronoi partitions, but the waypoints are randomly selected inside their own Voronoi cells. Finally, a fully-coordinated method is presented, where each robot tends to cover the area closer to its initial position, such that the robots movements are more organized. The trajectories of the robots under different methods during the mission are presented in Fig. 2(a), Fig. 2(b) and Fig. 2(c), respectively. From all these figures, the effectiveness and efficiency of the proposed coverage strategy can be verified.

\section{Conclusion}

In this paper, we proposed a coverage strategy for networked vacuum cleaner robots. Some preliminary results were obtained to validate the feasibility of the proposed method. For the next step of this research, we will implement the proposed algorithm on real robotic platforms and conduct real-world robotic experiments in large-scale complex environments.

\section{References}

1. Hu, J., Bhowmick, P., Lanzon, A.: Group coordinated control of networked mobile robots with applications to object transportation. IEEE Transactions on Vehicular Technology (2021)

2. Chen, J., Gauci, M., Li, W., Kolling, A., Groß, R.: Occlusion-based cooperative transport with a swarm of miniature mobile robots. IEEE Transactions on Robotics 31(2), 307-321 (2015)

3. Hu, J., Turgut, A.E., Lennox, B., Arvin, F.: Robust formation coordination of robot swarms with nonlinear dynamics and unknown disturbances: Design and experiments. IEEE Transactions on Circuits and Systems II: Express Briefs (2021) 

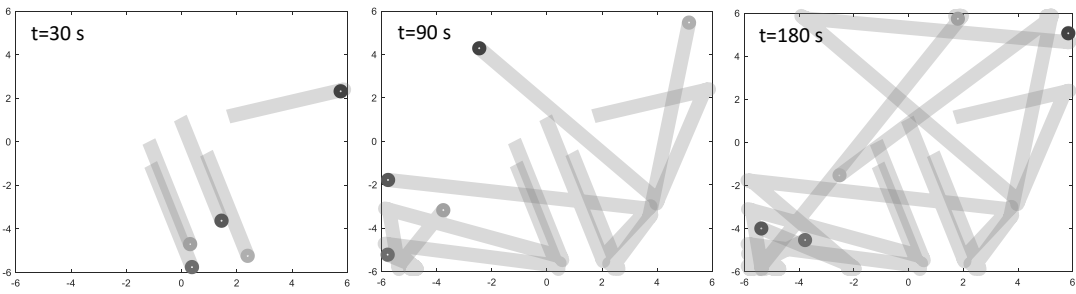

(a)
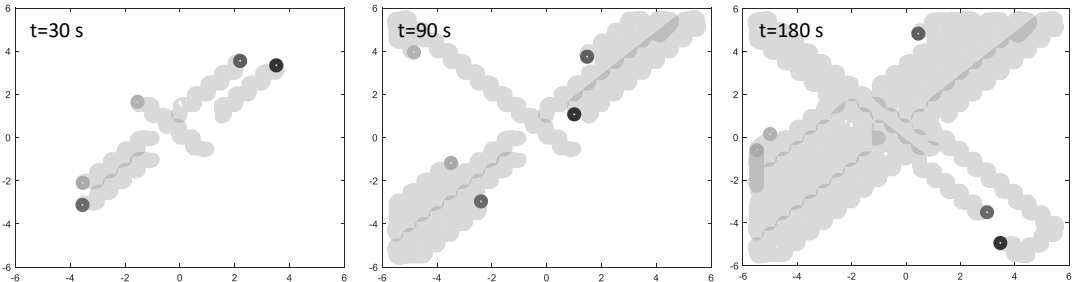

(b)
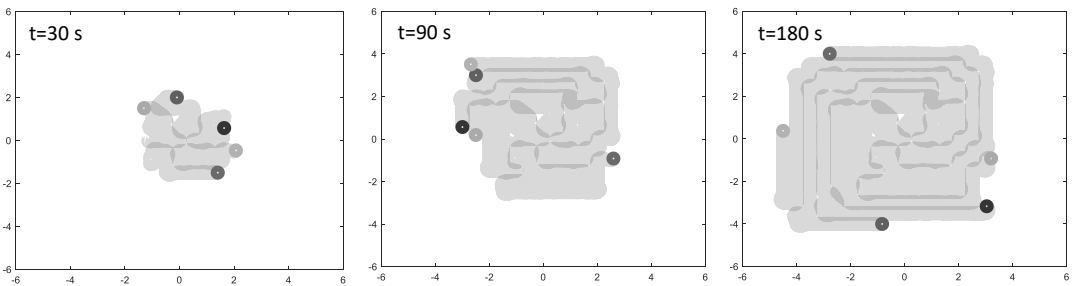

(c)

Fig. 2. Trajectories of the vacuum cleaner robots at different time instants. (a) Random movements. (b) Semi-coordinated coverage method. (c) Fully-coordinated coverage method.

4. Ban, Z., Hu, J., Lennox, B., Arvin, F.: Self-organised collision-free flocking mechanism in heterogeneous robot swarms. Mobile Networks and Applications (2021)

5. Keidar, M., Kaminka, G.A.: Efficient frontier detection for robot exploration. The International Journal of Robotics Research 33(2), 215-236 (2014)

6. Hu, J., Niu, H., Carrasco, J., Lennox, B., Arvin, F.: Voronoi-based multi-robot autonomous exploration in unknown environments via deep reinforcement learning. IEEE Transactions on Vehicular Technology 69(12), 14,413-14,423 (2020)

7. Ren, W., Sorensen, N.: Distributed coordination architecture for multi-robot formation control. Robotics and Autonomous Systems 56(4), 324-333 (2008)

8. Hu, J., Bhowmick, P., Jang, I., Arvin, F., Lanzon, A.: A decentralized cluster formation containment framework for multirobot systems. IEEE Transactions on Robotics (2021)

9. Michel, O.: Cyberbotics ltd. webots $^{\mathrm{TM}}$ : professional mobile robot simulation. International Journal of Advanced Robotic Systems 1(1), 5 (2004) 